\title{
Measuring the dynamics of contrast \& daylight variability in architecture: A proof-of-concept methodology
}

\author{
Siobhan Rockcastle*, Marilyne Andersen \\ Interdisciplinary Laboratory of Performance-Integrated Design (LIPID), School of Architecture, Civil and Environmental Engineering (ENAC), Ecole \\ Polytechnique Fédérale de Lausanne (EPFL), Lausanne, Switzerland
}

\section{A R T I C L E I N F O}

\section{Article history:}

Received 17 April 2014

Received in revised form

28 May 2014

Accepted 12 June 2014

Available online 25 June 2014

\section{Keywords:}

Daylighting design

Spatial contrast

Light variability

Visual effects

Dynamic lighting

Lighting perception

\begin{abstract}
A B S T R A C T
Unlike artificial light sources, which can be calibrated to meet a desired luminous effect regardless of latitude, climate, or time of day, daylight is a dynamic light source, which produces variable shadow patterns and fluctuating levels of brightness. While we know that perceptual impacts of daylight such as contrast and temporal variability are important factors in architectural design, we are left with an imbalanced set of performance indicators - and few, if any, which address the positive visual and temporal qualities of daylight from an occupant point-of-view. If visual characteristics of daylight, such as contrast and spatial compositions, can be objectively measured, we can contribute to a more holistic analysis of daylit architecture with metrics that complement existing illumination and comfort-based performance criteria. Using image processing techniques, this paper will propose a proof-of-concept methodology for quantifying contrast-based visual effects within renderings of daylit architecture. Two new metrics will be proposed; annual spatial contrast and annual luminance variability. Using 56 time-step instances (taken symmetrically from across the day and year) this paper will introduce a method for quantifying local contrast values within a set of rendered images and plot those instances over time to visualize hourly and seasonal fluctuations in contrast composition. Using the same 56 instances, this paper will also introduce a method for quantifying variations in luminance (brightness) between instances to measure fluctuations in brightness. This paper pre-validates each of the proposed methods by calculating annual spatial contrast and annual luminance variability across ten abstract digital models and comparing those results to the authors' own intuitive ranking.
\end{abstract}

() 2014 Elsevier Ltd. All rights reserved.

\section{Introduction}

Daylight offers both functional and aesthetic value to architecture, providing natural and energy-efficient illumination for interior tasks and infusing interior space with light, shadow, and texture. Unlike artificial light sources, which can be adjusted to meet a desired luminous effect regardless of latitude, climate, or time of day; daylight is sensitive to a number of dynamic conditions. The latitude of a given location affects the length and intensity of daylight hours across the year while climatic factors affect its strength and variability on an hourly scale. These variable conditions result in a highly dynamic source of illumination and perceptual phenomena. While many architects have expressed the importance of these phenomenological effects on our perception of

\footnotetext{
* Corresponding author.

E-mail addresses: Siobhan.rockcastle@epfl.ch, siobhan.rockcastle@gmail.com (S. Rockcastle), marilyne.andersen@epfl.ch (M. Andersen).
}

space [1-4] we are left with disproportionally few, if any, daylight design metrics that can evaluate the positive impacts of luminous variability within the visual field.

A preoccupation with electricity consumption, brought about by the oil crisis of the 1970s and strengthened through contemporary trends in energy conservation, encourages architects and engineers to place value in daylight as an energy-efficient alternative to artificial light. In an effort to reduce energy consumption, daylighting research has gravitated toward the widespread development of task-based illumination metrics [5] as a means of offsetting a building's reliance on electric light. Visual comfort metrics, especially those pertaining to glare, have also gained predominance within the last two decades, as the emphasis on daylight integration has led to an increase in glazed facades and complex shading systems that can trigger occupant discomfort during visual tasks. Advances in computational power have helped to facilitate time-intensive simulations, allowing us to transition from point-in-time glare risk-assessment to dynamic annual metrics $[6,7]$. Perceptual performance indicators such as contrast and 
variability, on the other hand, are traditionally defined as qualitative design factors and quantitative methods to explore their impact or relevance have been limited. Although subjective in nature, the perceptual performance of space is central to architectural design and often ranks above other more concrete evaluation criteria (like task-plane illuminance and visual comfort) within the design process. With this in mind, it is important to consider perceptual performance criteria alongside illumination and visual comfort metrics to develop a more holistic evaluation of daylight in architecture. A brief review of existing daylight performance metrics will help situate this paper and underline the need for new metrics that address the positive impacts of daylight within our field-of-view.

\subsection{Task-driven performance metrics}

The most common daylight metrics used today typically focus on task performance, whether in regard to illumination (workplane task illumination) or to visual comfort (eye-level glare evaluation). A third, less established category, but one of particular relevance to this paper, relates occupant preferences to perceptual factors such as brightness and luminous diversity within an established view position.

Over the past several decades, there have been significant improvements in our understanding of daylight as a dynamic and variable source of illumination. We have transitioned from static metrics such as Daylight Factor DF [8] to annual climate-based metrics like Daylight Autonomy DA [5] or Useful Daylight Illuminance UDI [9], as well as Acceptable Illuminance Extent AIE [10] when dealing with whole areas of interest over time, that all account for a more statistically accurate method of quantifying internal illuminance levels [11]. While DF may be the most widespread task-based illumination metric currently used in practice, it limits our understanding of daylight as a dynamic source of illumination, assuming a more-is-better attitude regardless of the sky type or intended programmatic use of the space under consideration [5]. Dynamic illumination metrics, such as DA, UDI or AIE, can evaluate annual illumination thresholds, taking into account building orientation and climate-driven sky type to provide a more accurate assessment of task-plane illuminance.

Unlike task-based illumination metrics that rely on illuminance, visual comfort metrics (typically pertaining to glare) tend to rely on luminance [12]. Of the four photometric quantities (luminous flux, intensity, illuminance, and luminance), luminance is most closely related to how the eye perceives light, and as such, appears as the only quantity capable of expressing visual discomfort. As luminance, brightness, and contrast are subjectively evaluated, methods to analyze glare discomfort are fragmented across no less than seven established metrics $[7,13,14]$. While these indices do not always agree, partly due to the fact that some were developed for electric lighting sources and others for daylight, most are derived from the same four quantities: luminance, size of glare sources, position of glare source, and the surrounding field of luminance that the eye must adapt to [15]. Glare-based visual comfort metrics, such as Daylight Glare Probability DGP [7], considered the most reliable index for side-lit office spaces under daylight conditions, have also evolved into dynamic annual metrics such as DGPs [15] which provides a comprehensive yearly analysis of glare, with limited computational intensity [6].

Task-driven illumination metrics such as DF and DA can be used to determine whether an interior space is sufficiently illuminated for the performance of visual tasks, whereas comfort-based luminance metrics such as DGP allow us to evaluate the visual field for sources of glare-based discomfort. While the shift toward climatebased metrics such as DA and UDI represents a significant improvement in daylight analysis, this data is limited to a twodimensional task-surface and does not correspond to the threedimensional view of space that is perceived by an occupant. Although dynamic glare metrics such as DGPs evaluate a threedimensional view position, they also only establish that high levels of contrast negatively impact visual comfort. Of the many established glare indices, not one addresses the notion of contrast as a positive visual effect.

Furthermore, task-driven illumination and visual comfort metrics are only applicable in spaces where visual tasks are frequently carried out. For spaces where visual tasks are less indicative of lighting performance, we have few, if any, broadly accepted metrics to help guide designers. In the absence of quantitative criteria, architects are tasked with creating acceptably bright or visually engaging environments, based on subjective criteria [16]. For many architects, this task is made difficult by the dynamic nature of sunlight and the challenges associated with predicting the range of visual effects that will occur across the day and year.

\subsection{Perceptual daylight metrics}

Two factors that are widely accepted to impact the field-of-view in daylit architecture are average luminance and luminance variation [17]. The former has been directly associated with perceived lightness and the latter with visual interest [18]. To evaluate the visual impacts of luminosity within interior architecture, existing research has relied on average luminance or "brightness," threshold luminance, and luminance variation (or standard deviation) in line with occupant surveys to establish trends in preference. Survey-based studies most commonly rely on high-dynamic-range HDR images, digital photographs or renderings produced through Radiance, which provide an expanded range of photometric information, allowing us to evaluate characteristics such as brightness and contrast $[19,20]$. Some studies have found that both mean luminance and luminance variation within an office environment contribute to occupant preference [21], whereas others have discovered that luminance distribution across an occupant's field-of-view [22] as well as the strength of variation are factors of preference [23].

In a study conducted by Cetegen et al., the authors discovered a positive trend between increased average luminance levels and satisfaction for the view in an office setting, but they also saw a trend between increased luminance diversity and the participant's impression of excitement [21]. In a second study, also conducted in an office setting, Tiller and Veitch concluded that a nonuniform lighting distribution increased an occupant's perception of brightness and preference [22]. Along the same lines, Wymelenberg \& Inanici conducted a study on occupant preferences toward light distribution in an office setting with horizontal blinds. The authors of the study concluded that adequate variations in luminance tended to create a stimulating visual environment, while excessive variability tended to create uncomfortable spaces [23].

The problem with those studies that rely on average luminance, luminance range, and standard deviation, is that they do not address the spatial diversity of luminance values within an occupants' field-of-view. In a daylight classification system proposed by Claude Demers, she categorizes digital images of architecture in terms of average brightness and standard deviation. While this method produces a typological language to codify lighting ambiance, she acknowledges that it cannot account for the spatial distribution of perceived light, which is central to the visual experience of architecture [24]. To address the importance of light distribution, Parpairi et al. developed the Luminance Difference (LD) index, which proposes a spatially dependent method for measuring luminance diversity across a selected view direction. 
This method relies on eye-level luminance measurements to calculate the difference in luminance levels across a range of acceptable angles corresponding to eye and head movement [25]. A study, which calculates the LD index across three selected view positions, found that luminance variability was highly appreciated by the participants and that variability rather than intensity were found to contribute to occupant satisfaction. While the LD index proposes a method for analyzing the spatial diversity of luminance values across an occupant's point-of-view, it does not address the temporal impacts of these visual effects. Furthermore, the method relies on physical measurements in live space, which can pose a number of practical problems, such as the movement of people and the disruption of equipment over extended studies.

In summary, existing research has produced promising indications that both luminance distribution and diversity play an important role in an occupant's perception of and preference toward the luminous environment. At the same time, we have yet to see an informative method for measuring the spatial diversity of luminance values across a human perspective, nor have we seen a method for quantifying the temporal impacts of daylight variability throughout the year. While most studies attempt to extract preference trends from luminance data, this paper seeks to establish a comparative framework and method for evaluating how contrasted or variable a scene may appear (over space and over time). While future research might explore the connection between contrast, variability, and human preferences toward light distribution, the authors believe that any evaluation of such perceived qualities must be based on specific design objectives. In other words, there are no definitive values for contrast or variability that necessarily indicate a 'good' or 'bad' set of visual performance criteria, but rather there might be a range of values that are recommended for the intended usage of a given space (such as a museum or office). These recommended values must be flexible enough to accommodate the designer's intent, while proving appropriate for the completion of any occupant tasks. As the composition of luminance is dynamic within daylit architecture, the authors of this paper will propose a new class of metrics that can quantify these perceptual effects over time. Building upon the author's thesis project at the Massachusettes Institute of Technology, this paper will introduce two new metrics; annual spatial contrast and annual luminance variability [44,45]. Using case study spaces to pre-validate results for each of the proposed metrics in a relative gradient from high to low, this paper will establish a methodology for comparing spatial contrast and luminance variability between spaces that are similar in size and orientation. As human preferences toward daylight are dependent on the intended program of an architectural space (i.e. its uses), this comparative framework will provide architects with a baseline against which design variations can be compared and perceptual qualities of daylight can be contextualized.

\section{Measuring contrast through digital images}

In image analysis, there are two types of measures that are commonly used to quantify contrast: those that rely on global measures and those that rely on local measures. Global measures most often rely on two single points of extreme brightness and darkness, taking into account the difference in maximum and minimum luminance values [26]. Other methods account for average luminance values, rather than extremes [27], and the standard deviation of image chroma (i.e. color channels) and lightness [28]. While these global contrast measures provide a single comprehensible value, they cannot effectively predict perceived contrast between two images that vary in composition [29].

Local contrast measures were developed to overcome the limitations associated with global measures by quantifying the effect of composition on contrasting areas of brightness and darkness. Included within this group of measures are methods that measure spatial frequencies in the Fourier domain [30], those that measure a weighted color contrast based on the distance between chroma regions [31], and those that calculate the difference between a single pixel and a surrounding region or neighborhood [32,33]. Despite this range of methods, there is still little consensus on how to produce a single number that represents the contrast perception of an image through localized pixel or neighborhood values [29]. On the one hand, a single number cannot easily distinguish between two images that vary in composition. This is due to the loss of information that occurs between a matrix of values or a power spectrum (resulting from a Fourier transformation) and the final value, which is often a mean or median value. On the other hand, a single number is a compact measure that can be compared to subjective experiments, which often produce a single value from occupant surveys.

For example, if we compare two renderings of daylit spaces sideby-side (Fig. 1) and relied on existing methods of global contrastanalysis, such as mean brightness or standard deviation, we would not be able to differentiate between the two images despite their varied compositions. The image on the left (Fig. 1a) shows a dense pattern of light and shadow - with a mean brightness of 120 and a standard deviation of 18 . The image on the right (Fig. 1b) contains larger patches of sunlight, but has a similar mean brightness of 132 and standard deviation of 22 . The obvious drawback to measurements like average luminance and standard deviation, is the loss of information that occurs when individual values are removed from the spatial framework of their composition.

As was discussed in Section 1.2, existing studies that address contrast perception within daylit architecture have focused on global measures such as standard deviation, luminance range, and average luminance, due to the ease of comparing single global measures against subjective rankings [23,24]. Given the lack of

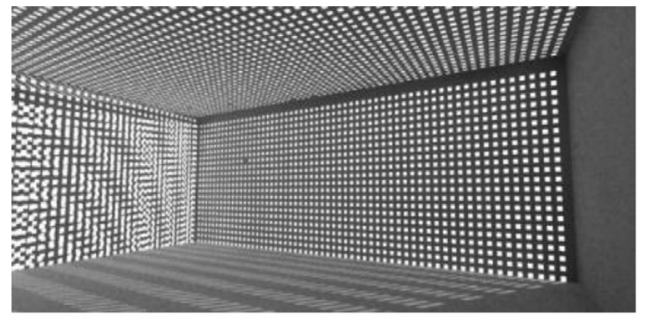

a. mean brightness: 120 , standard deviation: 18

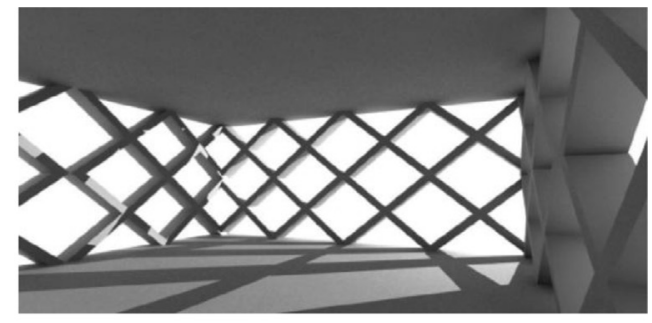

b. mean brightness: 132, standard deviation: 22

Fig. 1. Two abstract renderings that differ in composition, but share similar values for mean pixel brightness and standard deviation. 
Composition A

30 pixels: 15 black (0) and 15 white (255)

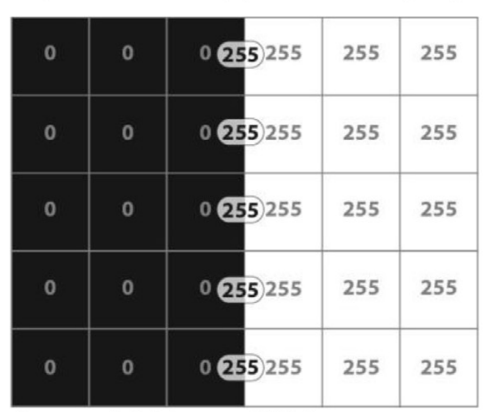

Average Brightness = 127.5

Standard Deviation $=129.7$

Spatial Contrast $\approx 10 \%$
Composition B

30 pixels: 15 black (0) and 15 white (255)

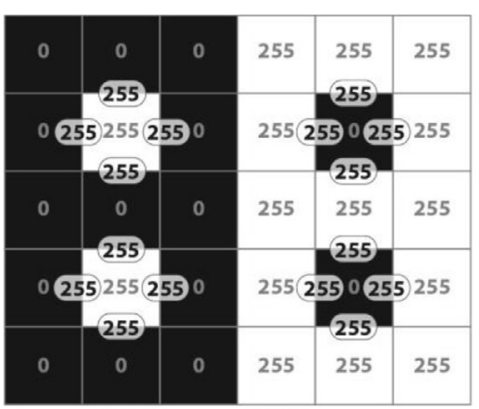

Average Brightness = 127.5

Standard Deviation $=129.7$

Spatial Contrast $\approx \mathbf{5 0} \%$
Composition C - MAXIMUM

30 pixels: 15 black $(0)$ and 15 white (255)

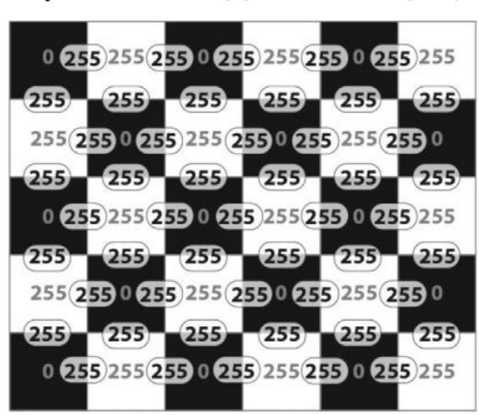

Average Brightness = 127.5

Standard Deviation $=129.7$

Spatial Contrast $=100 \%$

Fig. 2. A demonstration of spatial contrast in three compositions with the same number of black and white pixels, the same RGB average and standard deviation.

consensus over the use of global measures that accurately assess contrast perception in lighting design, contrast is often regarded as a qualitative element of daylight performance and is subjectively evaluated by designers through renderings or photographs that capture the luminous character of space. Although we can view an architectural rendering and describe the location and distribution of contrasting values within it, there are few methods that successfully quantify the spatial diversity of this distribution. Such a method could help designers to contextualize the relative strength of perceived contrast within an architectural space, use it to compare daylight-driven visual effects, and visualize how and when these effects vary over time.

\subsection{Quantifying spatial contrast}

In the context of this paper, the term Spatial Contrast will be used to describe the sum of local variations in brightness across the visual field, or - more specifically - the sum of variation between neighboring pixels within a rendering or photograph of daylit space. Drawing from an existing method of local contrast measure [33] which computes the difference between each pixel and its neighboring 8 pixels across various frequency levels, Fig. 2 illustrates the basic quantitative method proposed by this paper through a simple composition of black and white pixels. While luminance-based analysis methods often rely on HDR images due their dynamic range of pixel values, it is important that any imagebased contrast-analysis tool accommodate 8-bit images. This allows architects and educators to utilize a broader range of imagegeneration techniques; such as point-and-shoot photography and renderings from a range of geometric modeling platforms that may not accommodate HDR imaging. As such, we will use a standard pixel value range of RGB 0 (black) to RGB 255 (white) in this paper.

When composition $A$ is split down the middle, with half the pixels representing RGB 0 (black) and the other half representing RGB 255 (white), the average pixel brightness is 127.5, with a standard deviation of 129.7. Both average brightness and standard deviation are compact global measures used to describe luminance distribution in existing daylight research [21-24]. When, however, we rearrange the composition to create more perimeter between white and black pixels, such as can be seen in compositions B and C, the average pixel value and standard deviation remain unchanged despite the obvious increase in local contrast values.

To measure spatial contrast, we propose a method that calculates the difference in brightness between each pixel and its four neighboring pixels. While the final measure developed by Rizzi et al. [33] takes an average of local contrast values across the composition, our proposed measure computes the cumulative sum of local contrast values. We believe that the cumulative sum of local contrast values (and not the average) more accurately accounts for the compositional complexity of brightness and contrast across an image. As the cumulative sum can vary greatly between images that range in size, the final overall measure for spatial contrast is expressed as a ratio between the cumulative sum of local contrast values for a given image size $(n \times \mathrm{m})$ and a maximum hypothetical value based on the image size $(n \times m)$.

If we have an image matrix $P$ of size $(n \times m)$, each element $p$ contains a brightness value for a relevant pixel in the image composition. To calculate spatial contrast, the difference in brightness is taken between each pixel $p$ and its four neighboring pixels (column and row) and then averaged to produce a local contrast matrix of size $(n \times m)$ :

$$
\begin{aligned}
\overline{\Delta p_{i, j}}=\frac{1}{4} & \left(\left|p_{i, j}-p_{i+1, j}\right|+\left|p_{i, j}-p_{i-1, j}\right|+\left|p_{i, j}-p_{i, j+1}\right|\right. \\
& \left.+\left|p_{i, j}-p_{i, j-1}\right|\right)
\end{aligned}
$$

for all $i=1 \rightarrow n$ and $j=1 \rightarrow m$ (Fig. 3).

From these average local contrast values, spatial contrast can therefore be defined as:

$$
\text { Spatial Contrast }(\%)=\frac{\sum_{i=1}^{n} \sum_{j=1}^{m} \Delta p_{i, j}}{\Delta p \max _{i, j}} * 100
$$

where $\overline{\Delta p \max _{i, j}}$ is a hypothetical maximum value which is computed as a black and white checkerboard of size $(n \times \mathrm{m})$ where every pixel has an average local contrast of 255 :

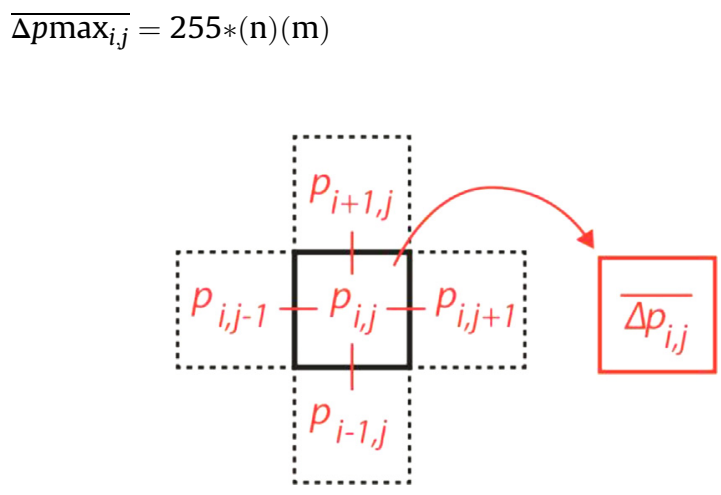

Fig. 3. The average difference between each pixel $p_{i, j}$ and its four neighboring pixels. 


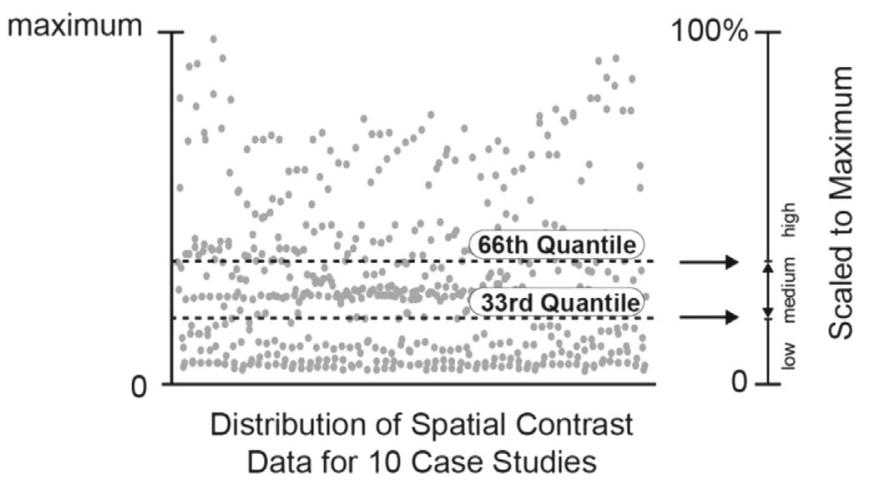

Fig. 4. All values for spatial contrast ( 56 time-steps $\times 10$ case study spaces $=560$ instances) have been normalized against the maximum value achieved through the case studies presented in Section 4.

As the hypothetical maximum value used to calculate spatial contrast is theoretically impossible for any architectural space to achieve, the resulting values must be adjusted to a range that is meaningful to differentiate them with as much resolution as possible. Fig. 4 shows the maximum value for spatial contrast achieved by the case study spaces that have been considered so far in this study and are introduced in Section 4, which happens to be $2.1 \%$. Within the scope of the present proof-of-concept study, this maximum value was then used to scale all remaining values from 0 to $100 \%$. Further development is needed to study the upper and lower thresholds of this scale for a broader set of architectural spaces, but the current range allows us to adequately compare the selected case studies.

When applying equations ( 1$)$ and (2) to the pair of $(584 \times 564)$ images introduced in Fig. 1, we can produce the corresponding spatial contrast $(583 \times 563)$ matrices illustrated through image form in Fig. $5 \mathrm{a}$ and b. As evidenced when compared to one another, their respective global and local contrast measures greatly differ in their ability to differentiate between images that vary in the spatial distribution and density of contrasting values. Fig. 5a shows an interior space with many small openings that emit highly articulated patterns of light and shadow, leading to a spatial contrast equal to $\approx 90 \%$. Fig. $5 \mathrm{~b}$, on the other hand, shows a rendering of a dia-grid façade with larger patches of light and shadow and a spatial contrast equal to $\approx 60 \%$. The distinguishably stronger spatial contrast in Fig. 5a, when compared to Fig. 5b thus corresponds to the intuitive differences in composition for these two examples much better than the global measures provided in Section 2 (mean brightness and standard deviation which were quite similar). This single example already shows the necessity to have complimentary local measures if one is to evaluate contrast in daylit architectural spaces. The next section builds upon these concepts by integrating the time component.

\subsection{An annual approach to spatial contrast}

In order to understand the impacts of daylight dynamics throughout the year, we applied the spatial contrast measure introduced in Section 2.1 to a series of time-stepped images to measure the cumulative effects of spatial contrast over time and visualize when and where these local variations occur throughout space. Using time-steps to divide the year into a series of moments, we can provide a representative cross-section of daylight-driven visual effects. We can then calculate the cumulative value of spatial contrast across these symmetrical annual instances and plot the results across a temporal map to visualize varied daily and seasonal impacts.

As the chosen time-step will influence the outcomes of any performance visualization, it is important to choose a resolution capable of describing climate and variations in sun course for a given location with sufficient detail. Towards this end, a method of climate based time segmentation originally developed for Lightsolve [34] will be used. Developed by Andersen and her research group, originally at MIT and now at EPFL, Lightsolve is a simulation platform that combines annual spatio-temporal maps with user-defined goals and associated annual daylight renderings for a climate-based analysis of daylight performance that splits the year into 56 representative time periods. It allows the designer to establish goal-based performance criteria and navigate the resulting maps of annual data alongside daylight renderings to provide a quantitative and simultaneously visual representation of performance in daylit space [10,35-38]. Its automated production of climate-driven annual renderings and temporal mapping capabilities make the method an ideal startingpoint for new image-based metrics that address perceptual factors of daylight performance on an annual scale.

The present paper adopts the same 56 periods supported by the Lightsolve method, but uses a CIE sunny sky model for all 56 renderings using the average sun position for the considered period. On the one hand, this simplification provides a consistent set of luminance maps that can be analyzed for relative changes in annual spatial contrast, and on the other hand, the resulting set of renderings can be used to represent an upper boundary for spatial contrast and variability as long as openings, depth, orientation, and positioning allow the limited set of 56 moments to reveal sun patches and adequate brightness. It should be noted that these 56 moments are being used as a starting-point for this proof-ofconcept study, but have not been validated in this context, only in climate-based analyses relying on illuminance calculations. To validate this annual time-step method in the next phase of research, the author's will compare the 56 periods proposed in this proof-of-concept study to a more frequent set of hourly calculations. Fig. 6 shows the 56 dates and legal times (Fig. 6b) for Boston, MA that are used to produce the annual set of renderings (Fig. 6a). The method for producing these renderings, including model geometry, building orientation, materials, and viewport setting will be described in more detail in Section 4 with the application of metrics to a series of case studies. The 56 instances listed in Fig. 6b

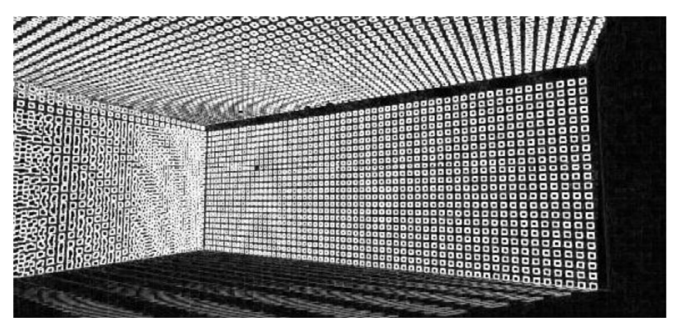

a.

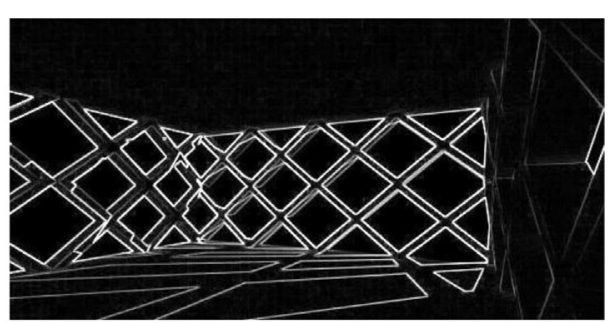

b.

Fig. 5. a. Spatial contrast equal to $\approx 90 \%$. b. Spatial contrast equal to $\approx 60 \%$. 


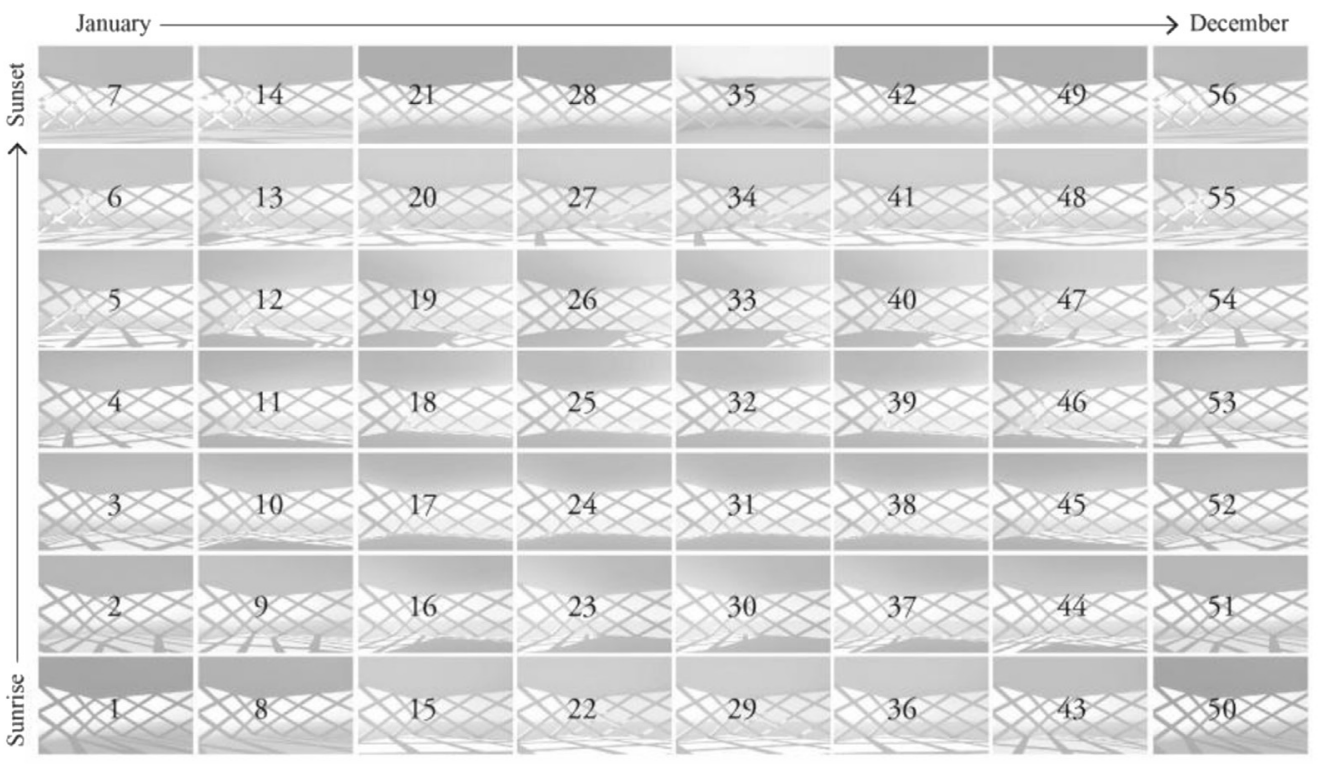

a.

\begin{tabular}{|c|c|c|c|c|c|}
\hline \multirow[t]{7}{*}{$\operatorname{Jan}-13$} & 1 & $07: 52$ & \multirow[t]{7}{*}{ Jul-15 } & 29 & $06: 25$ \\
\hline & 2 & $09: 12$ & & 30 & $08: 33$ \\
\hline & 3 & $10: 33$ & & 31 & $10: 41$ \\
\hline & 4 & $11: 53$ & & 32 & $12: 50$ \\
\hline & 5 & $13: 13$ & & 33 & $14: 58$ \\
\hline & 6 & $14: 34$ & & 34 & $17: 06$ \\
\hline & 7 & $15: 54$ & & 35 & $19: 14$ \\
\hline \multirow[t]{7}{*}{ Feb-28 } & 8 & 07:09 & \multirow[t]{7}{*}{ Aug-29 } & 36 & 07:03 \\
\hline & 9 & $08: 45$ & & 37 & $08: 57$ \\
\hline & 10 & $10: 21$ & & 38 & $10: 51$ \\
\hline & 11 & $11: 57$ & & 39 & $12: 45$ \\
\hline & 12 & $13: 33$ & & 40 & $14: 39$ \\
\hline & 13 & $15: 09$ & & 41 & $16: 33$ \\
\hline & 14 & $16: 45$ & & 42 & $18: 27$ \\
\hline \multirow[t]{7}{*}{ Apr-14 } & 15 & 07:03 & \multirow[t]{7}{*}{ Oct-14 } & 43 & $07: 43$ \\
\hline & 16 & $08: 57$ & & 44 & 09:18 \\
\hline & 17 & $10: 51$ & & 45 & $10: 54$ \\
\hline & 18 & $12: 45$ & & 46 & $12: 29$ \\
\hline & 19 & $14: 39$ & & 47 & $14: 04$ \\
\hline & 20 & $16: 33$ & & 48 & $15: 40$ \\
\hline & 21 & $18: 27$ & & 49 & $17: 15$ \\
\hline \multirow[t]{7}{*}{ May-30 } & 22 & $06: 15$ & \multirow[t]{7}{*}{ Nov-28 } & 50 & $07: 30$ \\
\hline & 23 & $08: 24$ & & 51 & $08: 50$ \\
\hline & 24 & $10: 33$ & & 52 & $10: 11$ \\
\hline & 25 & $12: 42$ & & 53 & $11: 32$ \\
\hline & 26 & $14: 50$ & & 54 & $12: 52$ \\
\hline & 27 & $16: 59$ & & 55 & $14: 23$ \\
\hline & 28 & 19:08 & & 56 & $15: 33$ \\
\hline
\end{tabular}

b.

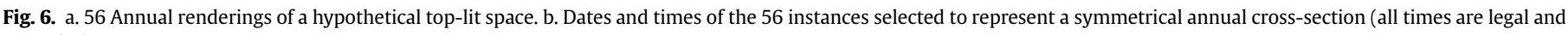
not solar).

will be used to produce all annual sets of renderings used for visual analysis in this paper.

In order to calculate annual spatial contrast, instantaneous spatial contrast is computed across all 56 moments shown in Fig. 6a. The cumulative sum of these instances, shown in Fig. 7a, highlights the location and strength of spatial contrast throughout the year, revealing areas that receive consistently high (red) and low (blue) contrast. In order to visualize when these dynamic effects of spatial contrast vary throughout the year, the sum of spatial contrast for each of the 56 renderings is plotted temporally to express the frequency and magnitude of daily and seasonal variations (Fig. 7b). The vertical axis of the temporal map shows daylight hours, from sunrise to sunset, while the horizontal axis shows days of the year, from January 1 st to December 31 st. Values are given a color scale to show relative strength from low to high, which has been adjusted to accommodate appropriate minimum and maximum values as determined by the case studies that will be introduced in Section 4 .

\section{Measuring variability in daylit space}

The second metric presented by this paper attempts to provide a measure of the annual variation in luminance values across a selected view. Whereas spatial contrast identifies compositional contrast boundaries between pixels within an image, and annual spatial contrast maps the accumulation of those contrast boundaries over time, annual luminance variability accounts for the cumulative difference in pixel values as they vary from one moment to the next. This metric is useful in describing the intensity of variation that occurs across a space as a combination of both time and dynamic natural lighting conditions. Many spaces that measure low in spatial contrast may still display moderate-tohigh amounts of luminance variability, as the brightness throughout a space can transform dramatically over the course of time while still maintaining a smooth compositional gradient in brightness.

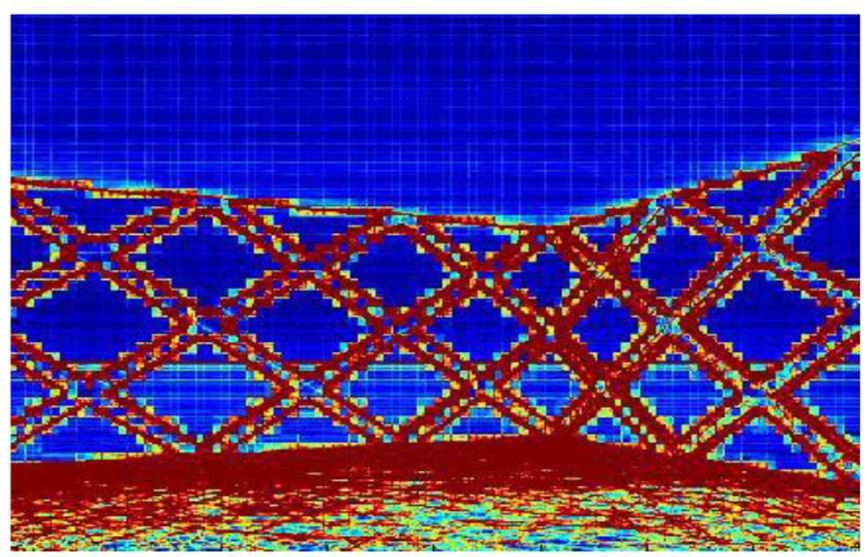

a.

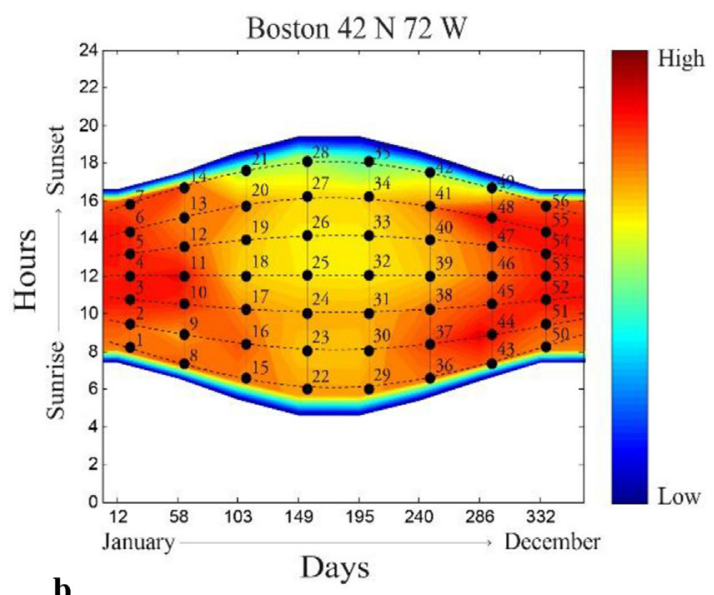

b.

Fig. 7. Annual spatial contrast results for a top-lit space. a. Cumulative annual map of luminance variability. b. Temporal map of luminance variability. 


\subsection{A quantitative approach to annual luminance variability}

To calculate luminance variability, this paper proposes a method to measure the variation in brightness of each pixel within an image as it varies between moments (or time-steps) due to dynamic climate and sun positions. When we use this method to quantify the variation in luminance levels on an annual scale, we must account for both daily and seasonal changes in the strength and dynamic orientation of sunlight. Its incident angle to the earth shifts hourly along a radial arc, altering its altitude and azimuth angle from sunrise to sunset, but the path of that arc also changes from one day to the next day. We must account for the difference between two moments that occur sequentially throughout the day as well as two moments that occur from one month to the next. As a first approximation, one can use the same time-step instances used to generate annual renderings for analysis in Section 2.2, illustrated in Fig. 8a. For each of these instances (we use 56 in this proof-ofconcept study, but there could be any number so long as they divide the year into symmetrical daily and monthly instances), luminance variability $\overline{\Delta P_{h, d}}$ can be calculated as the average difference between adjacent hourly and monthly instances, where each instance $P_{h, d}$ is composed of an image matrix $P$ at $h$ (hourly) and $d$ (daily) instance:

$$
\begin{aligned}
\overline{\Delta P_{h, d}}=\frac{1}{4} & \left(\left|P_{h, d}-P_{h+1, d}\right|+\left|P_{h, d}-P_{h-1, d}\right|+\left|P_{h, d}-P_{h, d+1}\right|\right. \\
& \left.+\left|P_{h, d}-P_{h, d-1}\right|\right)
\end{aligned}
$$

for all $P=1 \rightarrow n_{h}$ (number of hourly instances) and $j=1 \rightarrow n_{d}$ (number of daily instances).

Annual luminance variability is then calculated as the sum of all 56 instances divided by a hypothetical maximum sum of variation $\overline{\Delta P \max _{h, d}}$ :

Annual Luminance Variability $=(\%)=\frac{\sum_{h=1}^{n_{h}} \sum_{d=1}^{n_{d}} \overline{\Delta P_{h, d}}}{\overline{\Delta P \max _{h, d}}} * 100$

The hypothetical maximum $\overline{\Delta P \max _{h, d}}$ is determined by the same general principle as in $\overline{\Delta P_{h, d}}$, but where at each hourly and daily instance, the scene would theoretically alternate from fully

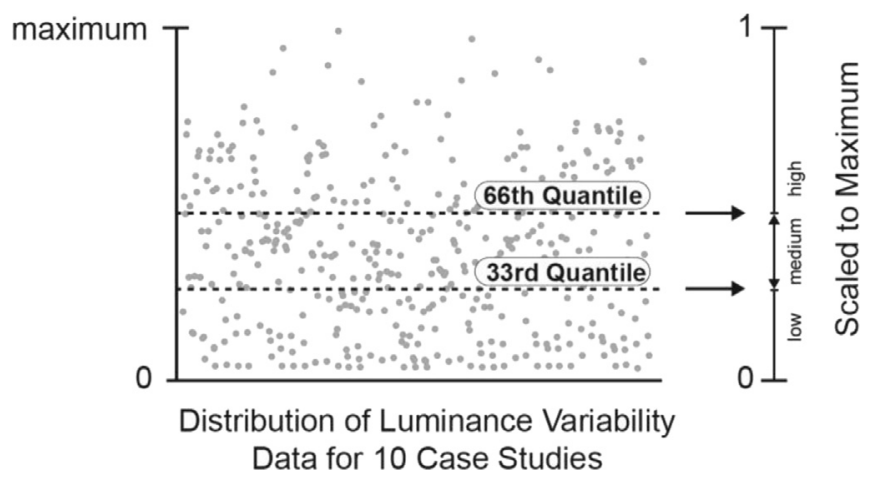

Fig. 9. Shows how all values for luminance variability have been normalized against the maximum value achieved through the case studies presented in Section 4.

white (255) to fully black (0) such that there is a maximum difference between each instance on both a "hourly" and "monthly" scale:

$\overline{\Delta P \max _{h, d}}=255\left(n_{h} \times n_{d}\right)(n \times m)$

To visualize how luminance variability changes over the year (i.e. when most variability occurs - under sunny sky conditions), a similar temporal map format as for spatial contrast can be used. When plotted temporally, these instances of luminance variability $\overline{\Delta P_{h, d}}$ can help to visualize when variations in luminance are the strongest across the year. While we decided not to take the difference between sunrise and sunset of any given day or the difference between December and January of the same year, future development of this method will explore the perceived variability that is observed at sunrise and sunset.

As for spatial contrast, instances of luminance variability have been normalized based on the maximum value achieved by the case studies introduced in Section 4. Fig. 9 shows that the maximum incremental value for luminance variability achieved by the case study spaces is 0.12 , which was then used to scale all remaining values from 0 to 1 in this proof-of-concept study.

Fig. 10 shows the results for annual luminance variability for a side-lit space (introduced in Fig. 1); it contains a cumulative spatial

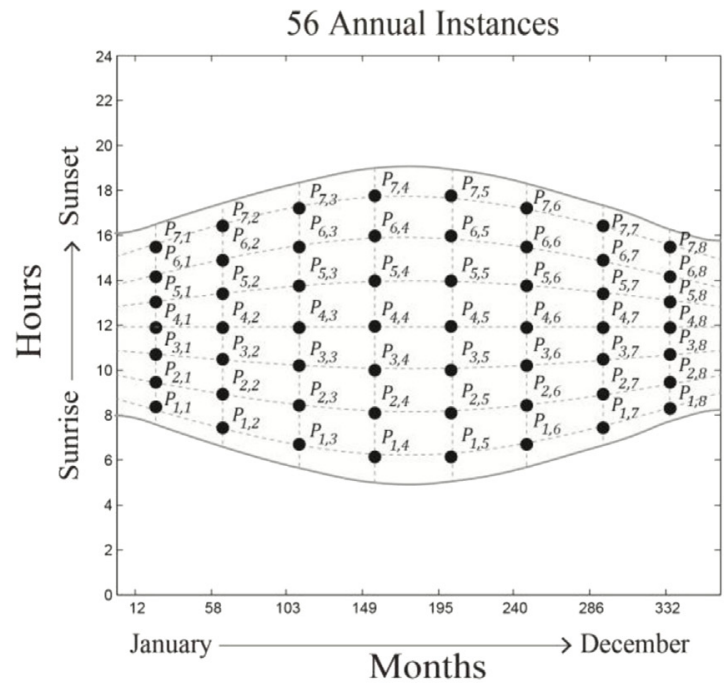

a.

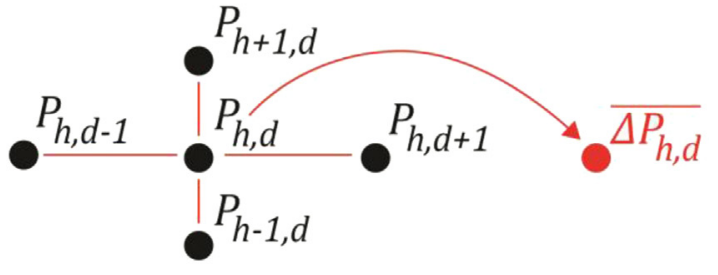

b.

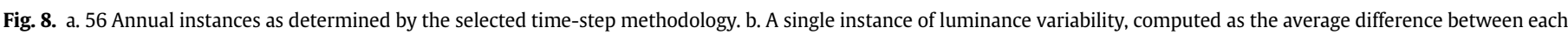
annual instance and its four neighboring instances (hourly and daily). 
matrix of variation across the whole year for the selected scene (Fig. 10a) and a spatio-temporal map that interpolates the 56 data points described in Section 3.1 (Fig. 8). This metric is useful in understanding where dynamic variations in natural light transform luminance levels across architectural spaces over the year (Fig. 10a) and when that variation is more abrupt or extreme (Fig. 10b). The temporal map in Fig. 10b shows that variations in luminance are most extreme in the winter when the sun is moving at a lower altitude, casting longer shadows into the interior. The color scale for this map will be introduced in Section 4.2, where results were conducted across a series of 10 case studies. The cumulative image in Fig. 9a shows where these variations occur within space, with changes appearing to be most extreme on the floor, as direct sunlight casts variable patterns of light and shadow through the diagrid façade and down into the space. Some changes can also be seen on the dia-grid, with minimum variation occurring across the ceiling, where light is (predictably) less variable than the horizontal surface of the floor.

Both annual spatial contrast and annual luminance variability account for distinct, yet related attributes of visual dynamics and help contribute to a more holistic understanding of architectural space as it is transformed by temporal shifts in sunlight across the day and year. The following section will apply these metrics to a set of typological case study models to display their analytical capabilities across a range of abstract architectural conditions.

\section{Evaluation of the metrics}

In order to evaluate these metrics across the year and determine whether they can adequately differentiate between a range of daylight conditions, 10 case study spaces were generated to represent an intuitive gradient of spatial contrast and luminance variability from high to low. Each space was modeled with the same generic floor plan and ceiling height dimensions as well as a fixed camera position to produce a comparable set of annual renderings. Each case study was modeled to emulate abstract conditions of existing interior architecture and represent a gradient of luminous effects. Although a more rigorous study of existing architectural spaces is required to validate the range of results for each metric, these 10 initial case studies allow us to establish relative thresholds for 'high' and 'low' spatial contrast and luminance variability.

\subsection{Selection \& modeling of case study spaces}

In order to select typological spaces that showcase a sufficiently broad range of daylight-driven visual effects, the authors conducted a survey of contemporary architecture. This survey contained 75 architectural spaces from around the world and was organized into 15 categories that describe the strength of spatial contrast and luminance variability intuited by the authors within each interior view. This matrix inspired the slightly-more-compact gradient of 10 case studies presented in this paper. The development of a typological ordering system for spatial contrast and luminance variability is presented elsewhere [39], but each case study was designed to resemble abstract characteristics found from within the larger matrix of existing architectural spaces. Fig. 11 shows the selected case studies in a line from 1 to 10 , representing the author's intuitive gradient of visual effects (before application of the metrics) from high spatial contrast and luminance variability on the left to low contrast and luminance variability on the right.

The annual spatial contrast and annual luminance variability metrics described in Section 3 were applied to this set of ten case study spaces. As a preliminary feasibility study, to verify whether the proposed metrics are capable of reproducing, through quantitative terms, a similar gradient to what the authors' intuition would establish. In order to compare these case studies, we first produced a set of annual renderings to describe the architectural qualities and daylight characteristics within each space.

Using Rhinoceros [40], each case study was modeled with the same floor area, ceiling height, and camera location so that results could be accurately compared (Fig. 12a). Cameras were positioned to face South, were centered in the East-West direction, and offset ten feet from the back wall to ensure an even distribution of wall, floor, and ceiling surfaces within each view (Fig. 12b). The Diva-forRhino toolbar [41] was then used to export the camera view to Radiance [42] with a vertical and horizontal viewport ratio set to $-\mathrm{vv} 40$ and $-\mathrm{vh} 60$. The specified materials were set to default reflectance values for floor, wall, and ceiling surfaces $(0.3,0.7,0.9$ respectively). The resolution of each image was rendered at high quality (a DIVA preset) to accommodate adequate detail with a $640 \times 480$ pixel aspect ratio (Fig. 12c). Although an individual rendering may be produced in Radiance using the DIVA toolbar, these proposed metrics require an automated set of 56 renderings for annual analysis. To automate this set, a Radiance batch script was used to generate a rendering for each date and time presented in Fig. 6b and Boston, Massachusetts was set as the location for all case study renderings (Latitude $42 \mathrm{~N}$, Longitude $72 \mathrm{~W}$ ). Although these metrics could eventually account for dominant sky conditions and evaluate the effects of climate on contrast and variability, we here focused exclusively on that the clearest comparisons, i.e. on sunny skies with direct light penetration. In order to analyze the potential impacts of contrast over time for this feasibility study, it

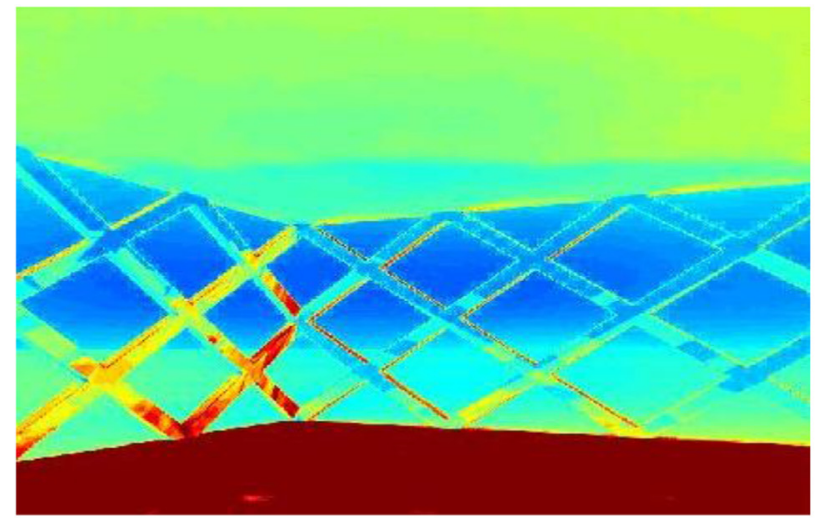

a.

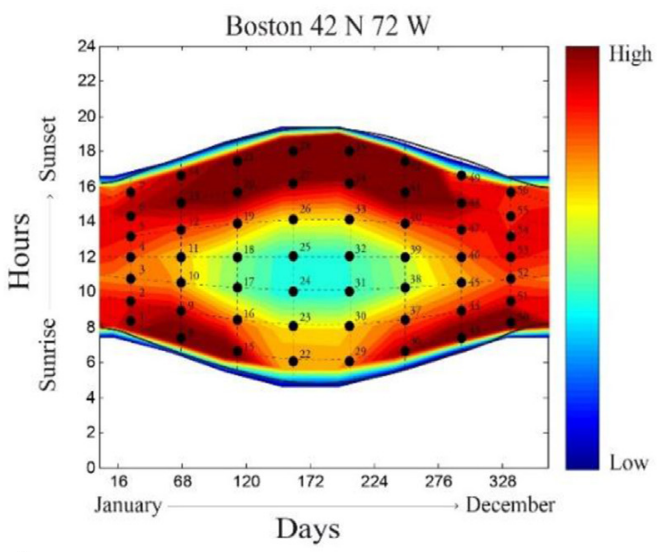

b.

Fig. 10. Annual luminance variability results for a side-lit space. a. Cumulative annual map of luminance variability. b. Temporal map of luminance variability. 


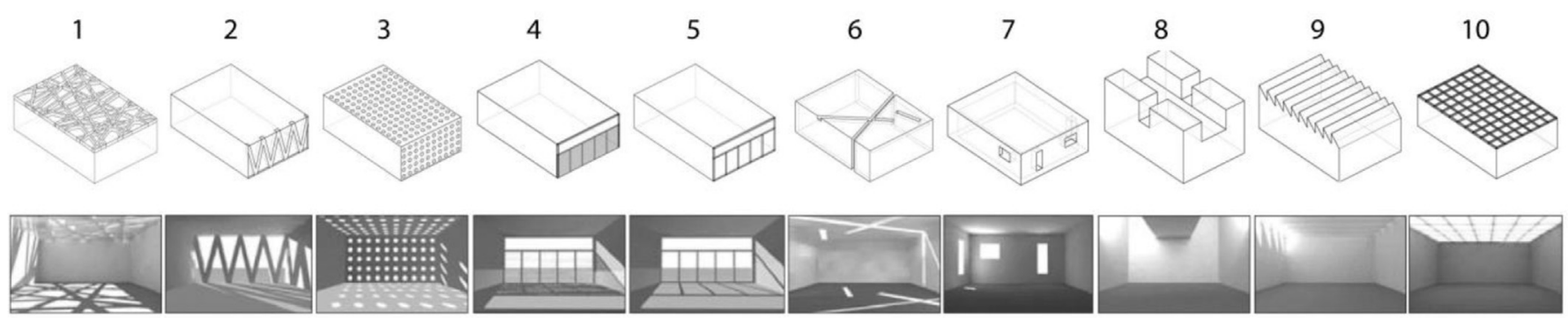

Fig. 11. Ten typological models from high contrast and variability on the left to low contrast and variability on the right.

was helpful to use a sky condition that allowed for maximized visual effects.

\subsection{Results for three case study models}

Annual spatial contrast and annual luminance variability is calculated for each set of radiance renderings produced using the method described in Section 4.2. The results of these metrics are discussed in the present section for three typological models that are representative of the whole range: case study one, four, and nine (Fig. 11).

The relative numerical scale for each metric (spatial contrast and luminance variability) has been determined by the results from all ten case studies using a statistical subdivision to generate a color scale of low, medium, and high values. Based on the distribution of resulting values, two thresholds divide the data for each metric into three parts, each representing a third of the population. A more comprehensive set of typologies will have to be analyzed in later stages of this research to validate and further refine this initial categorization. As a result of the statistical subdivision in this proofof-concept study, spatial contrast values between 0 and 0.25 are considered low, values between 0.25 and 0.4 are considered medium, and values exceeding 0.4 are considered high (Fig. 13a). Likewise, luminance variability values were split into three ranges, and values between 0 and 0.25 are considered low, values between 0.25 and 0.48 are considered medium, and values exceeding 0.48 are considered high (Fig. 13b). We will use these relative thresholds to discuss the results in terms of relative high, medium, and low, although future research is needed to develop a more statistically accurate range for each metric.

\subsubsection{Case study one}

Case study one was modeled to represent a highly contrasted and variable interior daylight environment with an open roof structure that casts dynamic and articulated patterns of light and shadow down onto the walls and floor. From the dynamic results presented in Fig. 14, case study one demonstrates a high degree of spatial contrast and luminance variability throughout the year. The temporal map in Fig. 14b shows a peak in spatial contrast between 10 a.m. and 3 p.m. in the summer months when the sun is directly overhead, while the temporal map for luminance variability in Fig. 14b shows maximum variations occurring through the same hourly window throughout the spring and fall. In Fig. 14c, thick red lines signify locations where spatial contrast was most consistent, highlighting the roof structure as the most redundant source with secondary accumulations on the floor and walls. Fig. 14e depicts the accumulation of luminance variation across all 56 images. Here, the floor is the area that experiences the most dramatic change over time. The cumulative effects shown in these two false-color images present an important distinction between metrics: Annual spatial contrast shows locations within the images where contrast occurs the most strongly and consistently, whereas annual luminance variability shows areas within the image where luminance is the most variable, emphasizing areas of dynamic instability. When compared side-by-side, these metrics allow us to discuss spatial contrast and temporal variability as related, but distinct visual characteristics present within the architectural space.

\subsubsection{Case study four}

Case study four represents a more traditional side-lit daylight strategy with a clerestory window above and louvered screen below, creating varied effects across the year depending on solar altitude (Fig. 15a). Here, the results for annual spatial contrast and luminance variability depict more seasonal variation, with a dramatic shift between the winter and summer months. The temporal map in Fig. 15b shows high spatial contrast between October and February, with moderate spatial contrast throughout the rest of the year. The location of these effects can be seen in the false-color image in Fig. 15c, which shows the accumulation of contrast on the walls and floor closest to the wall of louvers. Annual luminance

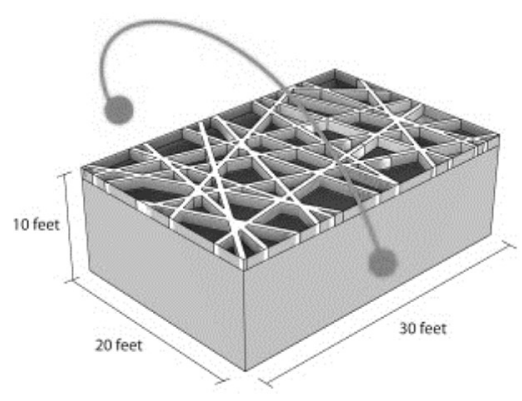

a.

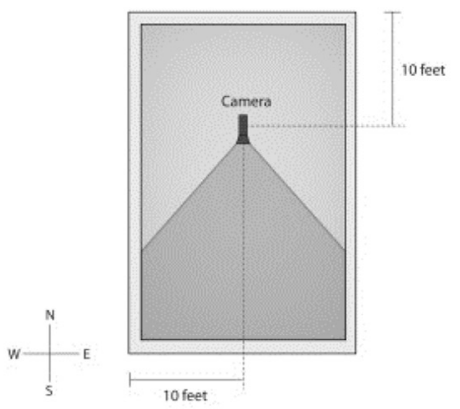

b.

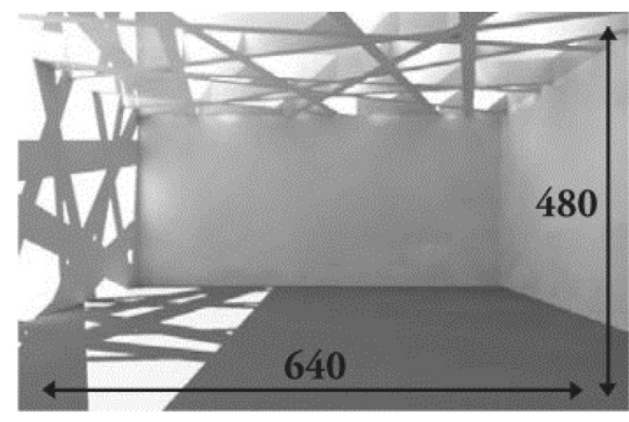

C.

Fig. 12. a) Exterior dimensions for each case study model, b) camera location in plan and c) sample image dimensions for HDR renderings. 


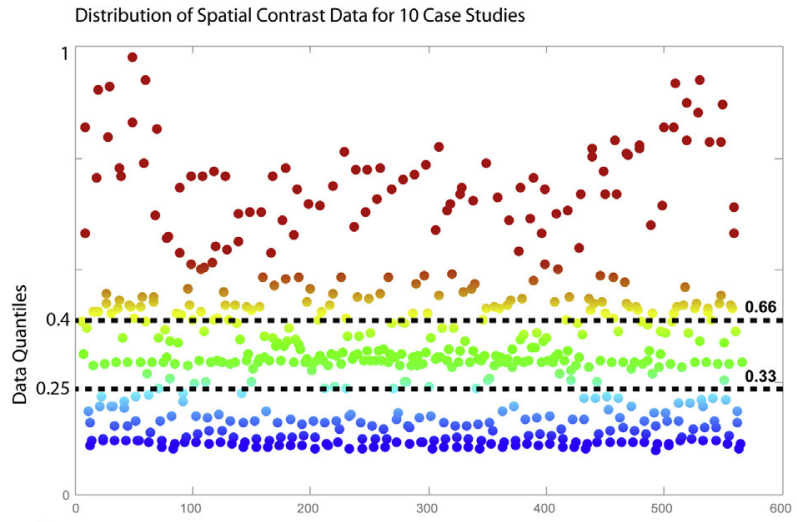

a.

Fig. 13. a) Distribution of spatial contrast data for 10 case studies and b) distribution of luminance variability data for 10 case studies.
Distribution of Luminance Variability Data for 10 Case Studies

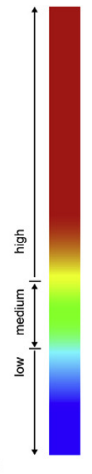

b.

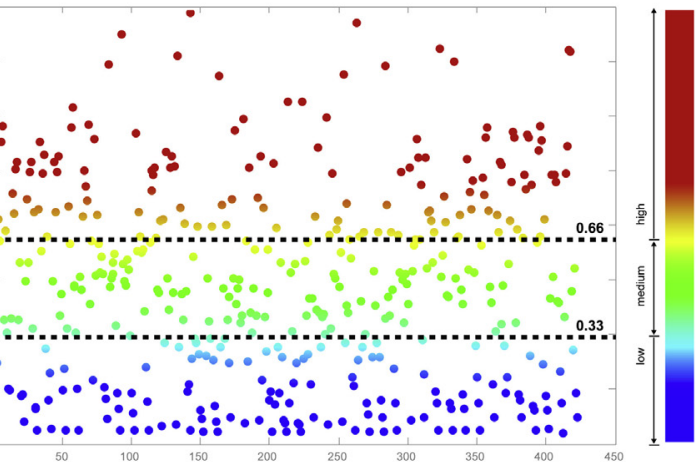

direct sunlight to penetrate the skylights and cast shadows across the walls and floor. While this causes minimal spatial contrast throughout the year, it does show a dramatic impact on luminance variability at sunrise and sunset throughout the spring and summer months. Fig. 16c shows the location of spatial contrast along the roof monitors, while Fig. 16e shows minimal luminance variability on the floor and walls, with a moderate degree occurring across the ceiling.

\subsection{Overview of ten case study results}

From this set of ten case study results, a clear gradient can be identified between the high and low ends of the contrast and variability spectrum. The three examples presented in Section 4.2 show dynamic variations in both spatial contrast and luminance variability as instantaneous values change over the course of the day and year. While it is clear from this proof-of-concept study that these measurements must be considered on a temporal scale due to their dynamic nature, it is also clear that each metric is distinct and
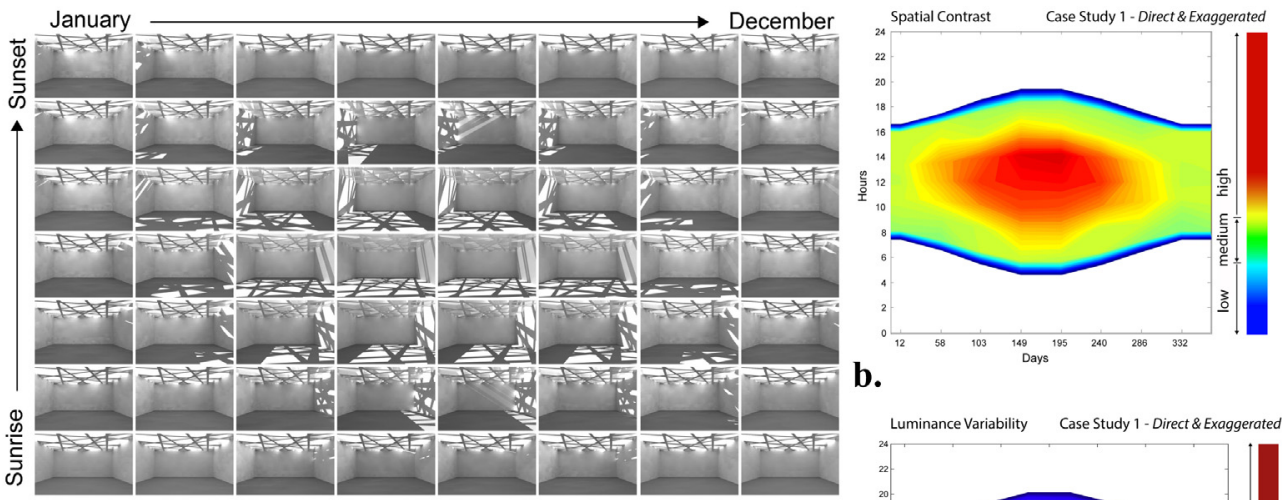

b.

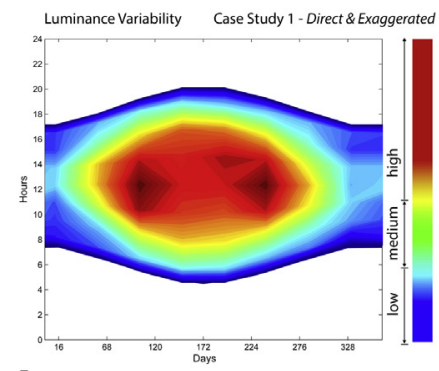

d.

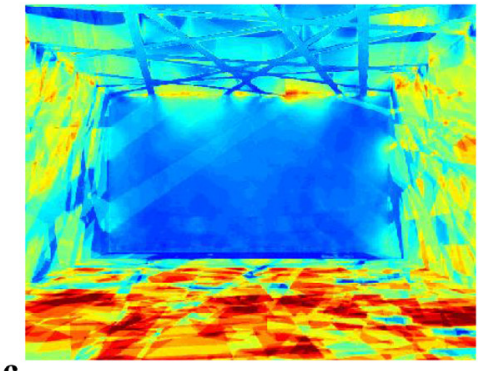

c.

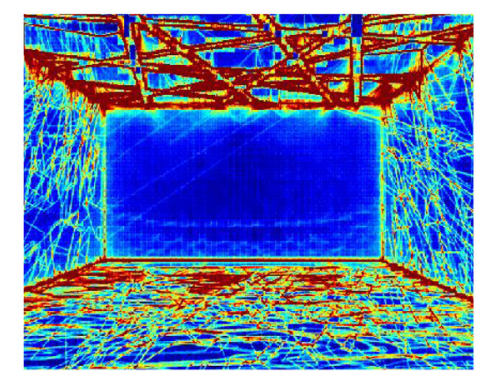

e.

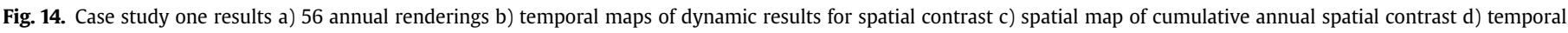
maps of dynamic results for luminance variability e) spatial map of cumulative annual luminance variability. 


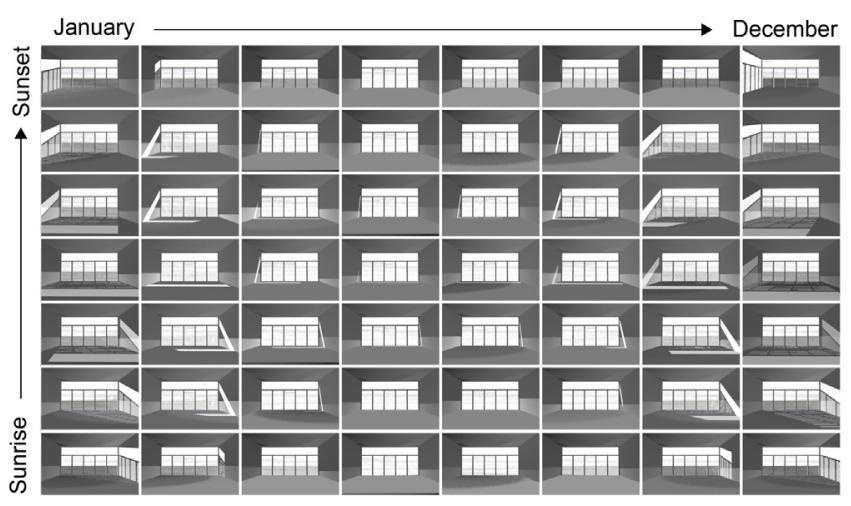

a.
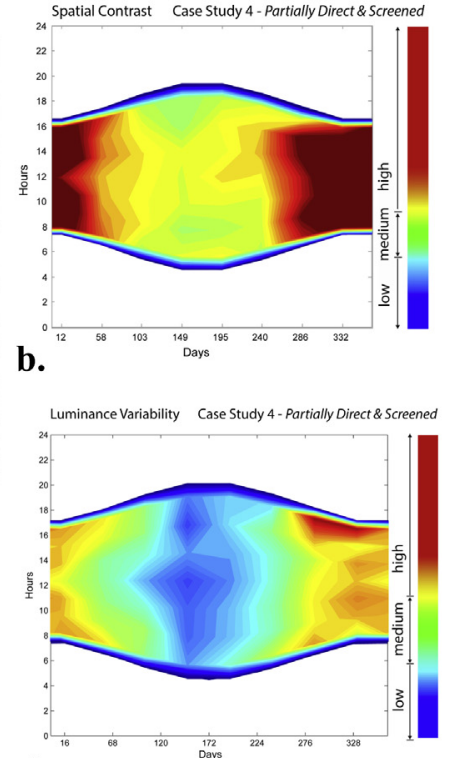

d.
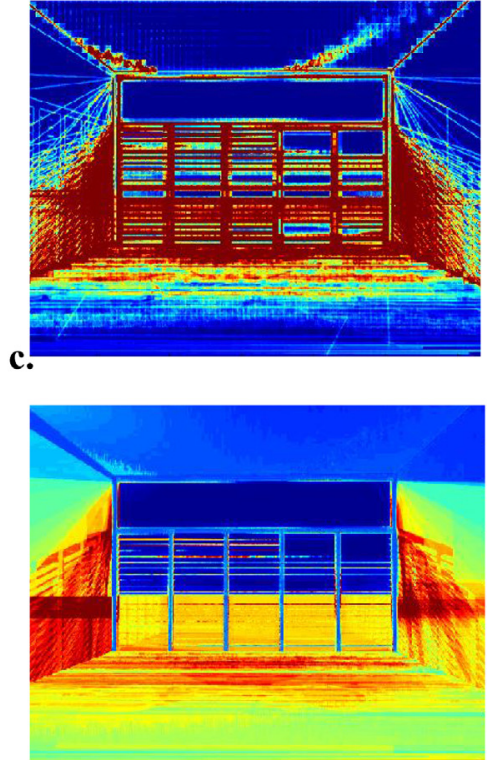

e.

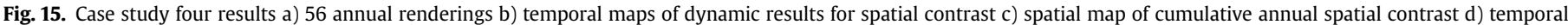
maps of dynamic results for luminance variability e) spatial map of cumulative annual luminance variability.

contributes to an alternate analysis of temporal conditions within architecture. In order to compare the results for each metric across the entire year, data for each of the 56 instances was converted into a single cumulative annual value. For annual spatial contrast, this number represents the sum of spatial contrast across all 56 instantaneous images. For annual luminance variability, this number represents a cumulative sum of luminance variability across each of the 56 moments; each instance representing the average difference between four neighboring moments (see Fig. 8b).

Fig. 17 shows normalized values (between 0 and the maximum cumulative value achieved across all ten case studies) for each annual metric. The resulting value for annual spatial contrast is described by the sum of instantaneous spatial contrast across all 56 instances, while annual luminance variability is described in Section 3.1. The dotted line shows the anticipated gradient of results based on the author's intuitive typological matrix, which was introduced in Fig. 11. This comparison between quantitative measure and intuitive typological matrix is helpful in determining whether the proposed measures agree with the subjective intuition of the authors. As a proof-of-concept study, this helps to confirm the sensitivity of the proposed measures in differentiating between spaces that were intuited, subjectively by the authors, to vary in terms of contrast composition and variability over time. Future developments of this method will require a more rigorous

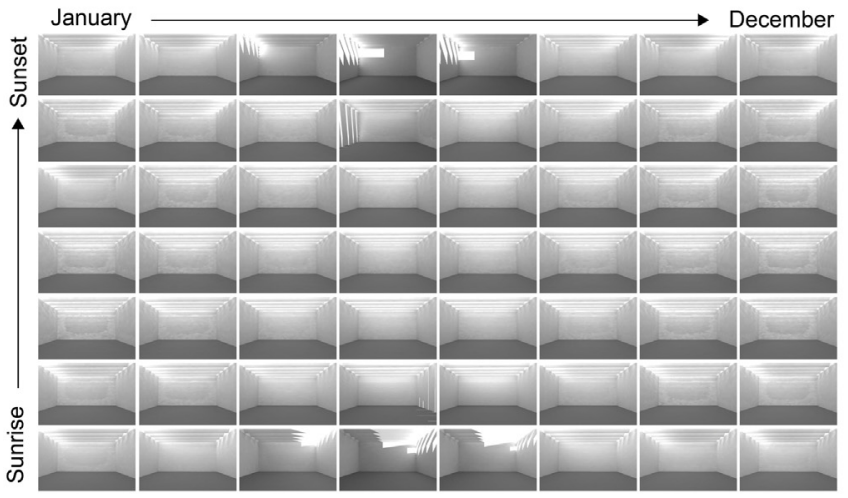

a.
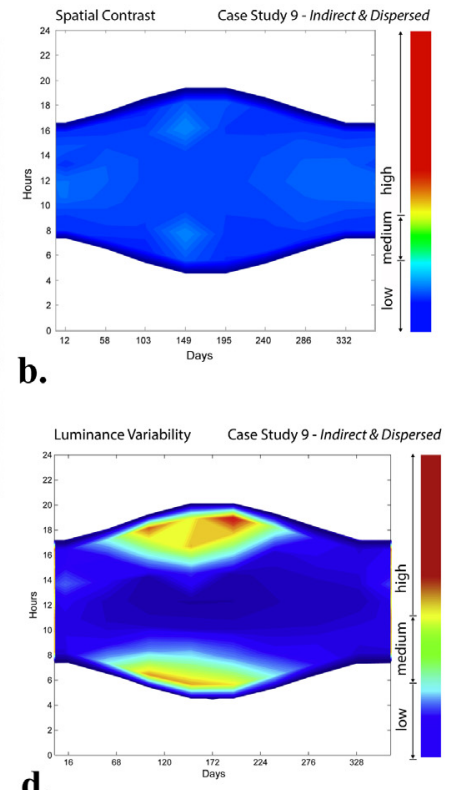
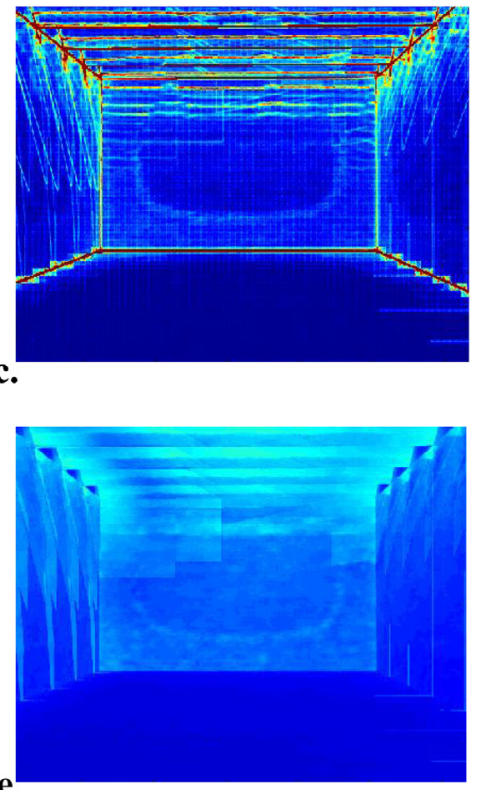

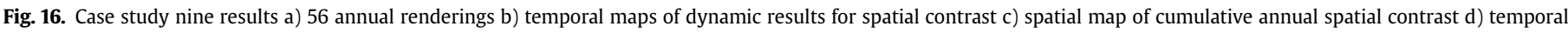
maps of dynamic results for luminance variability e) spatial map of cumulative annual luminance variability. 


\section{Annual Spatial Contrast \& Luminance Variability}

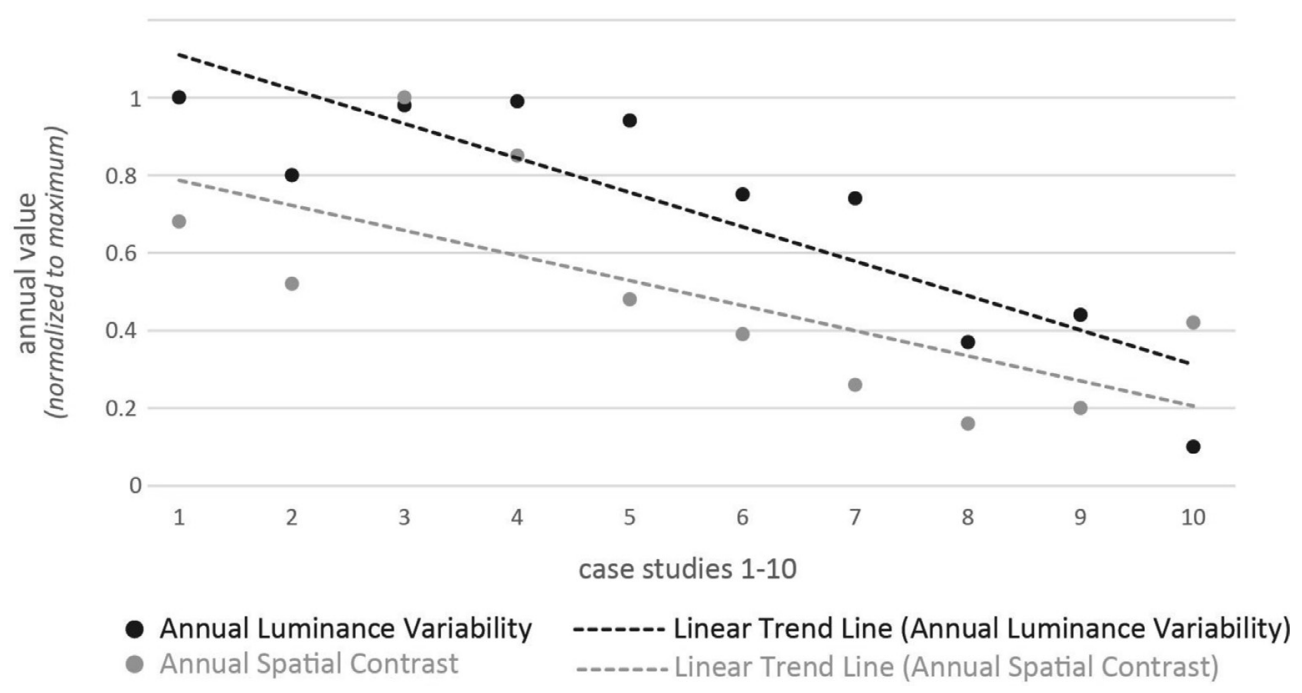

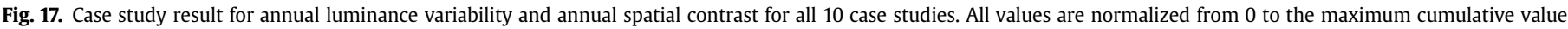
across all spaces.

validation to prove that the authors' intuitive ranking is consistent with a larger sample of human subjects and a larger and more varied sample of architectural spaces.

While case studies one and ten break the trend for annual spatial contrast, they fit the trend for annual luminance variability, resulting in the highest and lowest accumulative annual values (respectively). Case study two, on the other hand, appear to be an outlier for both annual metrics. In retrospect, the authors agree that case study two was located in the wrong relative position within the intuitive gradient and that it should have been located closer to the middle of the matrix, between case studies five and six. The authors predicted that the dia-grid geometry would produce more dominant shadow patterns within the interior, while in actuality the results were quite similar to the side-lit office space in case study five. The only difference between case studies two and five is, of course, the specific geometry of openings, but they produce similar amounts of contrast and variability within the interior.

If we consider the temporal map of instantaneous results, the proposed metrics reveal surprising changes in luminance and contrast in case study nine. These changes were difficult to anticipate due to the geometry of the roof and incident solar angles, and we believe that these isolated discrepancies between intuition and the annual measures further exemplifies the importance of dynamic visual analysis methods. In this case, the metrics helped to reveal perceptual changes within the visual field that were difficult to anticipate by the experts due to the dynamic nature of sunlight over time. If our intuition is unable to 'predict' the variation in contrast at different times of the day or year, than these annual metrics can help designers to make objective decisions in the selection of various design scenarios.

This proof-of-concept study allows for the relative comparison between each of the ten case study spaces using the proposed metrics for spatial contrast and luminance variability. For example, we can say that the louvered space in case study six represents a relatively high degree of both annual spatial contrast and annual luminance variability, while the screened space in case study four shows a high degree of annual spatial contrast with a low degree of annual luminance variability. These numbers also allow us to speculate that case study one experiences more annual luminance variability than any other space within the study. Although the proposed measures for each metric require further development, these pre-validation results demonstrate the capabilities of annual spatial contrast and annual luminance variability in quantifying a relative set of qualitative temporal characteristics within daylit architecture.

\section{Discussion \& future work}

This research raises an important set of issues for architects and daylight designers. How do we leverage perceptual performance indicators against those task-based illumination and visual comfort metrics that dominate the field of daylight performance analysis? The application of these proposed measures for contrast and light variability to the ten case studies introduced in Section 3 was an attempt to link an intuitive and subjective assessment of perceptual effects to a quantitative measure. To further develop and validate these new image-based measures, we have identified two phases of future research. The first phase will expand the current set of case studies to include a more extensive range of detailed architectural models. This expanded set of examples will allow us to test the sensitivity of the proposed measures against a broader database and further calibrate the scale for each metric (which is currently based on the results achieved by the ten case studies). The second phase will conduct an experimental study to validate (through a statistically significant population size) the relationship between human perceptions of contrast and temporal variability and the proposed measures, which have only been discussed through the authors' trained, but limited, subjective assessment. This experimental study will use renderings from the examples produced in phase one to construct a gradient of contrast perception based on the participants' subjective impressions. This gradient will then be compared to existing measures for contrast, brightness, threshold luminance, luminance diversity, and proposed metrics for spatial contrast.

Another area of future work includes the refinement of quantitative measures proposed by this paper. The current method takes an accumulative difference between neighboring pixels to produce a set of boundary conditions within a given image. Although this 
accounts for a fine level of detail in local luminance variation, the measure is dependent on pixel density and does not account for larger regions of contrast that may be perceived by the human eye. A broader look into the cognitive sciences and digital image processing techniques may produce a more holistic approach to quantifying spatial contrast. Furthermore, the selection of 56 annual time-steps must be studied to determine whether they represent an adequate frequency to measure the effects of contrast and variability on a dynamic scale. While this method has been validated for task-based illumination metrics, it has not been validated in this context, and must be compared to an hourly time-step calculation to find an appropriate frequency of hourly and daily instances.

Additionally, it would be useful to consider the impacts of color on the perception of contrast and perceptual dynamics in daylit architecture. In a study conducted by Simone et al., they concluded that colored images were often rated higher in perceived contrast than their greyscale counterparts [43]. As the color temperature of daylight varies due to sky conditions, orientation, and time of day, it would be interesting to consider the influence of color and brightness on our perception of contrast and light variability.

Ultimately, it is important to propose the integration of these metrics into a software package so that perceptual performance may be measured alongside task-based illumination, visual comfort, and health-based metrics to provide a more holistic evaluation of daylit space. The Lightsolve project, created at MIT and currently under development at EPFL, proposes an adaptation of these metrics alongside non-visual and dynamic comfort metrics as part of an integrated tool to assess human needs in daylit architecture [35]. Through an integration task-based metrics for illumination and visual comfort, photobiological metrics for health, and perceptual field-of-view metrics like spatial contrast and luminance variability, the designer will be able to fine-tune his/her analysis to fit individualized performance criteria specific to climate, architectural program, and design intent.

\section{Conclusion}

This paper began with a review of existing daylight analysis metrics to establish the need for new performance criteria that can account for the range of perceptual and temporal qualities found within architectural space. These qualities, if measureable, could then be positioned alongside existing task-based illuminance and visual comfort metrics to provide a more holistic analysis of daylight performance in architecture. To contextualize these perceptual and temporal qualities within the discipline of architecture, the authors conducted a survey and produced a typological matrix of daylighting design strategies based on an intuitive assessment of each space [39]. This typological study revealed a diverse range of daylight-driven visual effects and was used to formalize a set of qualities within each space that contributed to the authors' subjective assessment. Using this intuitive matrix as context, this paper then proposed two new metrics: annual spatial contrast and annual luminance variability which attempt to measure the effects of spatial and temporal diversity of daylight in architecture through time-stepped renderings.

In order to measure the intuitive effects described above, HDR renderings were used to record luminance levels within a selected view and then compressed down into 8-bit images, providing a compact range of values that could be analyzed. Although spatial contrast looks at the variation between neighboring pixel values within a selected image, annual spatial contrast and luminance variability account for the dynamics of contrast and variations in brightness throughout the year. Through an analysis of an annual set of images ( 56 renderings that represent an even subdivision of daylit hours across the year; 7 daily and 8 monthly) the designer can identify the magnitude of spatial contrast and luminance variability over time and visualize these dynamic effects through a combination of accumulative spatial images and annual temporal maps. When applied to the ten case study spaces introduced in Section 4, each proposed measure produced a trend in relation to the author's intuitive ranking, which serves as a pre-validation in this proof-of-concept study. When compared to existing daylight performance metrics such as daylight factor, daylight autonomy, and daylight glare probability; these new annual, image-based metrics provide important quantitative information about the dynamic perceptual effects of daylight that have been previously unexplored.

These new annual metrics communicate information about the spatial and temporal quality of daylit, providing architects with a tool for comparing the magnitude of visual effects within architecture. The implications of this work are widespread, from a simple analytical tool for describing dynamic daylight conditions, to an objective approach that challenges the use of task-based illumination and visual comfort metrics in a variety of programmatic conditions. By establishing an intuitive gradient of visual effects and producing a method for quantifying those effects over time, we are able to re-focus the discussion on daylight performance to include those perceptual qualities of light that are often disregarded in contemporary practice.

\section{References}

[1] Holl S. Luminosity/porosity. Tokyo: Toto; 2006.

[2] Zumthor P. Atmospheres: architectural environments - surrounding objects Basel: Birkhäuser; 2006.

[3] Pallasmaa J. The eyes of the skin: architecture and the senses. West Sussex: Wiley \& Sons; 2012.

[4] Steane MA, Steemers K. Environmental diversity in architecture. New York: Spoon Press; 2004.

[5] Reinhart C, Mardaljevic JRZ. Dynamic daylight performance metrics for sustainable building design. Leukos 2006;3(1):1-25.

[6] Jakubiec J, Reinhart C. The 'Adaptive Glare Zone' - a concept for assessing discomfort glare through daylit spaces. Light Res Technol 2012;44(2):149-70.

[7] Wienold J, Christofferson J. Evaluating methods and development of a new glare prediction model for 152 daylight environments with the use of CCD cameras. Energy Build 2006;38(7):743-57.

[8] Moon P, Spencer D. Illumination for a non-uniform sky. Illum Eng 1942;37(10):797-826.

[9] Nabil A, Mardaljevic J. The useful daylight illuminance paradigm: a replacement for daylight factors. Energy Build 2006;38(7):905-13.

[10] Kleindienst S, Andersen M. Comprehensive annual daylight design through a goal-based approach. Build Res Inform 2012;40(2):154-73.

[11] Mardaljevic J. Simulation of annual daylighting profiles for internal illuminance. Light Res Technol 2000;32(3):111-8.

[12] CIE. Commission Internationale de l'Eclairage Proceedings. Cambridge: Cambridge University Press; 1926.

[13] IESNA. IESNA lighting handbook: reference and application. New York: Illuminating Engineering Society of North America; 2000.

[14] Osterhaus W. Discomfort glare assessment and prevention for daylight applications in office environments. Sol Energy 2005;79(2):140-58.

[15] Wienold J. Dynamic daylight glare evaluation. In: Proceedings of Internationa IBPSA Conference, Glasgow; 2009.

[16] Cuttle C. Towards the third stage of the lighting profession. Light Res Technol 2010;42(1):73-93.

[17] Veitch J, Newsham G. Preferred luminous conditions in open plan offices: research amd practice recommendations. Light Res Technol 2000;32(4): $199-212$.

[18] Loe D, Mansfield K, Rowlands E. Appearance of lit environment and its relevance in lighting design: experimental study. Light Res Technol 1994;26(3): 119-33.

[19] Ward G. The RADIANCE lighting simulation and rendering system. In: Proceedings of '94 SIGGRAPH Conference, Orlando; 1994.

[20] Newsham G, Marchand R, Veitch J. Preferred surface luminance in offices: a pilot study. In: Proceedings of the IESNA annual conference, Salt Lake City; 2002.

[21] Cetegen D, Veitch J, Newsham G. View size and office illuminance effects on employee satisfaction. In: Proceedings of Balkan light, Ljubljana, Slovenia: 2008.

[22] Tiller D, Veitch J. Perceived room brightness: pilot study on the effect of luminance distribution. Light Res Technol 1995;27(2):93-101. 
[23] Wymelenberg K, Inanici M. A study of luminance distribution patterns and occupant preference in daylit offices. In: PLEA2009-26th conference on passive and low energy architecture, Quebec City; 2009.

[24] Demers C. A classification of daylighting qualities based on contrast and brightness analysis. In: Conference proceedings of the American Solar Energy Society (ASES), Cleveland; 2007.

[25] Parpairi K, Baker N, Steemers K, Compagnon R. The luminance differences index: a new indicator of user preferences in daylit spaces. Light Res Technol 2002;34(1):53-68.

[26] Michelson A. Studies in optics. Chicagp: University of Chicago Press; 1927.

[27] King-Smith P, Kulikowski J. Pattern and Flicker detection analysed by threshold summation. J Physiol 1975;249(3):519-48.

[28] Calabria A, Fairchild M. Perceived image contrast and observer preference II. Empirical modelling of perceived image contrast and observer preference data. J Imaging Sci Technol 2003;47:494-508.

[29] Simone G, Pedersen M, Hardeberg JY. Measuring perceptual contrast in digital images. J Vis Commun Image R 2012;23(3):491-506.

[30] Hess R, Bradley A, Piotrowski L. Contrast-coding in amblyopia. I. Differences in the neural basis of human amblyopia. In: Proceedings of Royal Society of London series B (217), London; 1983.

[31] Tremeau A. Color contrast parameters for analysing image differences. In: Proceedings of the University of Derby Color Image Science 2000 conference, Derby; 2000.

[32] Tadmor Y, Tolhurst D. Calculating the contrasts that retinal ganglion cells and LGN neurones encounter in natural scenes. Vision Res 2000;40(22):3145-57.

[33] Rizzi A, Algeri G, Medeghini D, Marini A. A proposal for contrast measure in digital images. In: Second European conference on color in graphics, imaging and vision, Aachen; 2004
[34] Kleindienst S, Bodart M, Andersen M. Graphical representation of climate based daylight performance to support architectural design. LEUKOS 2008;5(1):39-61.

[35] Andersen M, Guillemin A, Amundadottir M, Rockcastle S. Beyond illumination: an interactive simulation framework for non-visual and perceptual aspects of daylighting performance. In: Proceedings of IBPSA 2013, Chambéry; 2013.

[36] Andersen M, Gagne J, Kleindienst S. Interactive expert support for early stage full-year daylighting design: a user's perspective on Lightsolve. Automation Constr 2013;35:338-52.

[37] Gagne J, Andersen M, Norford L. An interactive expert system for daylighting design exploration. Build Environ 2011;46(11):2351-64.

[38] Andersen M, Kleindienst S, Yi L, Lee J, Bodart M, Cutler B. An intuitive daylighintg performance analysis and optimization approach. Build Res Inform 2008;36(6):593-607.

[39] Rockcastle S, Andersen M. Celebrating contrast and daylight varibaility in contemporary architectural design: a typological approach. In: Proceedings to LUX EUROPA, Krakow, Poland; 2013.

[40] [Online] http://www.rhino3d.com 2007. [accessed 2010]

[41] [Online] http://www.diva-for-rhino.com 2009.

[42] [Online] http://www.radiance-online.org/ 2009.

[43] Simone G, Pederson M, Hardeberg J. Measuring perceptual contrast in uncontrolled environments. In: Proceedings of the 2nd European workshop on visual information processing, Paris; 2010.

[44] Rockcastle S, Andersen M. Dynamic annual metrics for contrast in daylit architecture. In: SimAUD conference proceedings, Orlando; 2012.

[45] Rockcastle S, Andersen M. Annual dynamics of daylight variability and contrast: a simulation-based approach to quantifying visual effects in architecture. Springer, London: Springer Briefs in Computer Science; 2013. 